\title{
Antibiotika-Anwendung 2012/13 in 109 deutschen Akutkrankenhäusern
}

\author{
W. V. Kern ${ }^{1}$, M. Fellhauer ${ }^{2}$, M. Hug ${ }^{1}$, T. Hoppe-Tichy ${ }^{3}$, G. Först ${ }^{1}$, M. Steib-Bauert ${ }^{1}$, K. de With ${ }^{4}$
}

\section{Zusammenfassung}

Hintergrund | Mit der zunehmenden bakteriellen Resistenz-Entwicklung und dem Anstieg der Clostridium-difficile-Infektionen wird die Sicherstellung einer rationalen und intelligenten Antibiotikaverordnung immer wichtiger. Voraussetzung hierfür sind auch zuverlässige Daten zur Antibiotikaverschreibung. Bisher waren solche Daten für Akutkrankenhäuser in Deutschland nicht ausreichend verfügbar.

Methode I Wir analysierten die Antibiotikaverordnungsmengen aus 109 Akutkrankenhäusern über einen Zeitraum von je einem Jahr (2012/13) in Bezug auf Pflegetage, Krankenhausgröße und bestimmte Fachabteilungen. Die Darstellung erfolgte deskriptiv in Tagesdosen pro 100 Pflegetage, wobei die Tagesdosisdefinition speziell für den Krankenhausbereich adaptiert wurde (,recommended daily dose“, RDD).Zusätzlich wurden aber auch die seitens der WHO definierten Tagesdosen („defined daily dose“, DDD) berechnet.

Ergebnisse I Die Antibiotika-Verbrauchsdichte betrug insgesamt 43,5 RDD/100 Pflegetage (Median) mit einem Interquartilbereich von 3648 RDD/100 (entsprechend 64,4 DDD/100 [53,3$73,9])$. Die Verbrauchsdichte war in Universitätskliniken signifikant höher als in den sonstigen Krankenhäusern, wohingegen keine Unterschiede zwischen den nicht-universitären Krankenhäusern unterschiedlicher Größe beobachtet wurden.

Auf Intensivstationen war die Verordnungsdichte rund doppelt so hoch wie auf den Normalstationen. Insgesamt machten die Verordnungen auf Intensivstationen jedoch lediglich $12 \%$ (nicht-universitäre Krankenhäuser) und 18\% (Universitätskliniken) des gesamten Antibiotikaverbrauchs aus.
Sehr hohe Verordnungsdichten wurden auch in universitären hämatologisch-onkologischen $\mathrm{Ab}$ teilungen beobachtet. Cephalosporine wurden etwas häufiger als Penicilline eingesetzt. Fluorchinolone machten nach Cephalosporinen und Penicillinen die dritthäufigste Substanzklasse aus. Der Anteil von Cephalosporinen der ersten und zweiten Generation und der dritten und vierten Generation am Gesamtverbrauch je Krankenhaus schwankte von $5 \%$ bis $37 \%$ bzw. von $<1 \%$ bis $29 \%$. Die fünf entsprechend ihren Tagesdosen am häufigsten eingesetzten Einzelsubstanzen waren:

- Cefuroxim,

- Piperacillin-Tazobactam,

- Ceftriaxon,

- Metronidazol und

- Ciprofloxacin.

Folgerung I Die Intensität der Verordnung systemischer Antibiotika in Akutkrankenhäusern in Deutschland ist mit im Mittel $>40$ Tagesdosen pro 100 Pflegetage hoch, zeigt aber eine starke Variabilität je nach Fachabteilung und Substanz/ Substanzklasse. Bemerkenswert häufig wurden Cephalosporine verordnet. Inwieweit diese Befunde mit unterschiedlichen Risiken der ResistenzEntwicklung und Clostridium-difficile-Infektionsraten zusammenhängen, ist nicht klar.

Weitere Erfassungen und Analysen der Antibiotikaverbrauchsmuster in Akutkliniken wie diese könntem zusammen mit Rückmeldung und fachbereichsorientiertem Benchmarking wichtige Anstöße zur Optimierung des Antibiotika-Einsatzes sowie zur Planung und Beurteilung von Strategien zur Resistenzminimierung, Eindämmung von Clostridium difficile-Infektionen und verbesserten Patientensicherheit geben.

\section{Einleitung}

Die Intensität der Antibiotika-Anwendung ist über den damit ausgeübten Selektionsdruck auf Erreger wie auch auf Mikroorganismen der Begleitflora wesentlich für die bakterielle ResistenzEntwicklung [1,2]. Die Veränderungen in der bakteriellen Mikroflora nach Anwendung von Antibiotika sind erheblich. Sie persistieren oft Wochen bis Monate nach Behandlungsende. Seit mehreren Jahren ist man daher bemüht, Quantität und Qualität der Antibiotikaverschreibung sowohl in der Humanmedizin als auch in der Veterinärmedizin und Lebensmittelproduktion zu erfassen. Es soll geprüft werden, ob und wie hier Verbesserungspotenziale ausgeschöpft werden könnten, mit denen Resistenz-Entwicklung minimiert werden kann. Eine entsprechende Erfassung von Ver-
Institute

1 Universitätsklinikum Freiburg

2 Schwarzwald-Baar-Klinikum, Villingen-Schwenningen

3 Universitätsklinikum Heidelberg

4 Universitätsklinikum Carl Gustav Carus, Dresden

\section{Korrespondenz}

Prof. Dr. Winfried V. Kern Abteilung Infektiologie Klinik für Innere Medizin II Universitätsklinikum Freiburg Hugstetter Straße 55 79106 Freiburg Fon 0761 270-18190 Fax $0761270-18200$ kern@if-freiburg.de 


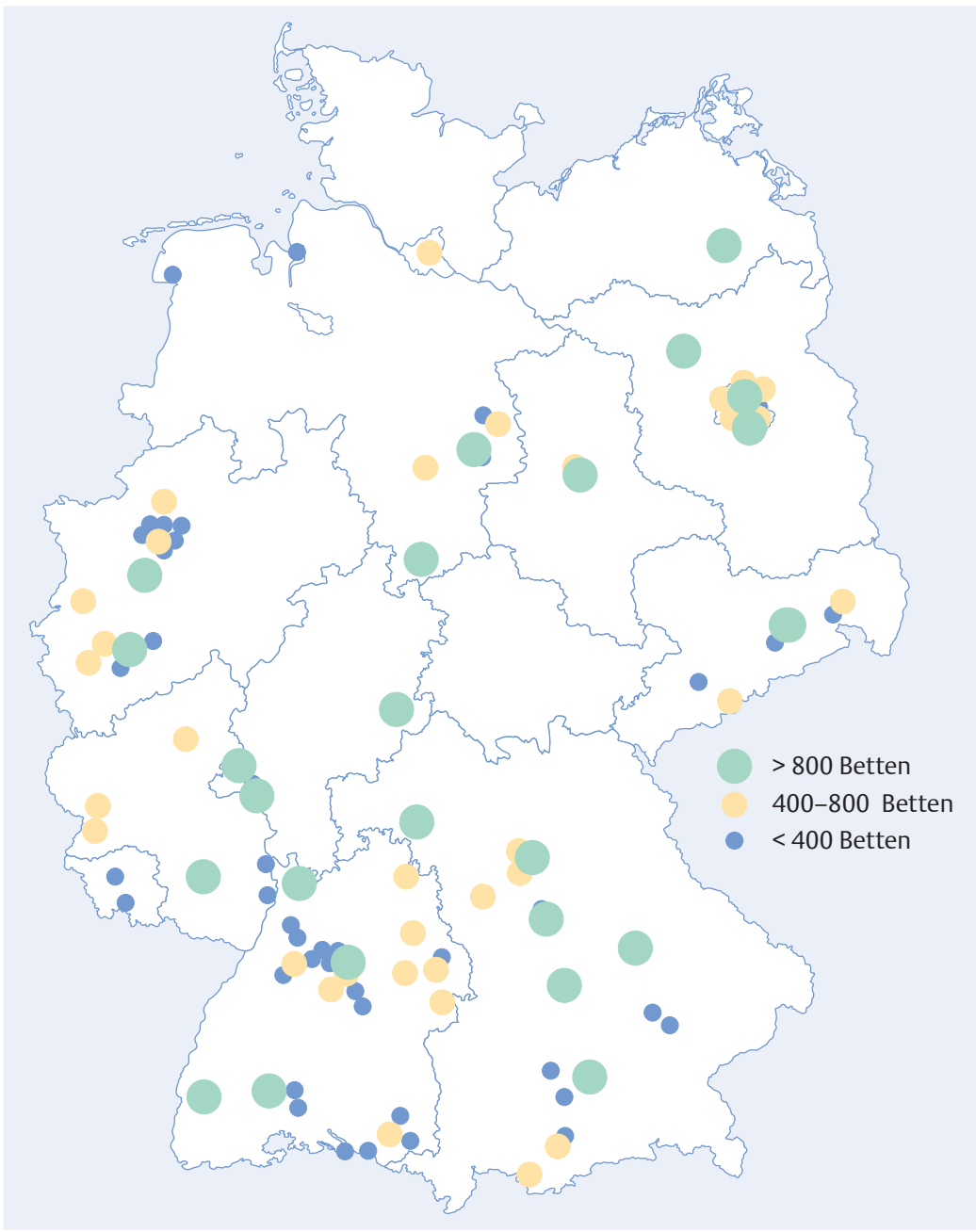

Abb. 1 Geografische Lage der in der Erhebung in den Jahren 2012/13 beteiligten Akutkrankenhäuser. brauchsmengen und -mustern ist für die ambulante Medizin in vielen Ländern bereits zur Routine geworden [3]. Im Bereich der Veterinärmedizin sind ebenfalls Fortschritte erzielt worden [4].

In Deutschland gibt es zu beiden Bereichen inzwischen relativ gute Verbrauchsmengenstatistiken, mit denen Detail-Analysen möglich und gezielte Interventionen zur Qualitätsverbesserung planbar geworden sind. Die Entwicklung der Verbrauchsmengen hierzulande wird unter anderem zusammen mit der Entwicklung der AntibiotikaResistenz in den so genannten GERMAP-Berichten gemeinsam für Human- und Veterinärmedizin dargestellt [5-7] - nach dem Vorbild der dänischen DANMAP-Reporte, die es dort schon seit 1996 gibt [8].

Für den Krankenhausbereich hat sich in nahezu allen größeren europäischen und außereuropäischen Ländern eine Erfassung des Antibiotikaverbrauchs bisher schwierig gestaltet [3, 9, 10]. Der Anteil der im Krankenhaus verordneten Antibiotika an der Gesamtmenge ist zwar relativ klein, die Antibiotika-Verbrauchsdichte in Akutkrankenhäusern ist jedoch sehr hoch. Der entsprechend hohe Selektionsdruck betrifft hier zudem in erster Linie die Mikroflora von Kranken und teilweise erheblich infektionsgefährdeten Patienten und wird durch erhöhte Transmission von Mikroorganismen zwischen Kranken kompliziert $[11,12]$.

Damit befindet sich die Krankenhausmedizin in einem besonderen Spannungsfeld: Es sollen modernste Behandlungsverfahren einschließlich Organersatz und Transplantation sowie Onkologie und Intensivmedizin auf höchstem Niveau angeboten werden, diese kommen aber alle nicht ohne hocheffektive Antibiotikaverschreibung aus. Um dem damit erhöhten Risiko der Selektion und Übertragung von resistenten Erregern mit anschließenden manifesten Infektionen zu begegnen, müssen alle erdenklichen Möglichkeiten der Minimierung von Antibiotika-Resistenzproblemen einschließlich der Optimierung der Arzneimittelsicherheit und Verordnungsqualität genutzt werden.

Eine sorgfältige und sachgerechte Verbrauchsmengenerfassung unter Berücksichtigung der zahlreichen Einflussfaktoren wird damit essenziell. So können Anwendungsmuster und deren Folgen für die Resistenz-Entwicklung erkennbar gemacht und für den Krankenhausbereich optimierte Antibiotikastrategien identifiziert werden. Dies stellt die Voraussetzung für eine nachhaltige Qualitätssicherung dar, was so auch in der neuen deutsch-österreichischen S3-Leitlinie zur Sicherung rationaler Antiinfektivaverordnung im Krankenhaus [13] und in vielen anderen Leitlinien und Empfehlungen [14, 15] gefordert wird.

In der vorliegenden Arbeit haben wir aktuelle Daten zum Antibiotikaverbrauch aus 109 deutschen Akutkrankenhäusern analysiert. Ziel ist die Darstellung von Benchmarks, Größenordnungen und Verbrauchsmustern für Antibiotika unter Berücksichtigung von Krankenhausgröße und diversen Fachdisziplinen sowie intensivmedizinischen Bereichen und Normalstationen. Diese Informationen sind von Bedeutung für Infektions- und Hygienekommissionen, Arzneimittelkommissionen und Qualitätsmanagementabteilungen. Sie können und sollen bei der Planung weiterer Aktivitäten zur Verbesserung der Antibiotikaverordnungsqualität in Akutkliniken helfen. Das verwendete Erfassungs- und Berichtssystem bietet Flexibilität für die Bearbeitung lokaler krankenhausspezifischer Fragen und zugleich enorme Perspektiven in der Versorgungsforschung zum Thema optimierte Antibiotikastrategie, ResistenzEntwicklung und Patientensicherheit.

\section{Methoden}

Die Daten stammen aus Akutkliniken (Allgemeinkrankenhäuser), die am so genannten ADKA-ifRKI-Projekt (www.antiinfektiva-surveillance.de) 


\begin{tabular}{|lll|lll|}
\hline & \multicolumn{3}{c}{ RDD*/100 } & \multicolumn{2}{c|}{ DDD*100 } \\
\hline Klinikgröße/-art & $\mathbf{n}$ & Median & $\begin{array}{l}\text { Interquartil- } \\
\text { bereich }\end{array}$ & $\begin{array}{l}\text { Median } \\
\text { Interquartil- } \\
\text { bereich }\end{array}$ \\
\hline Nicht-universitäre Krankenhäuser & 98 & & & & \\
\hline <400 Betten & 50 & 40,1 & $34,7-47,6$ & 61,0 & $51,3-71,8$ \\
\hline $\mathbf{4 0 0 - 8 0 0 ~ B e t t e n ~}$ & 34 & 42,3 & $35,1-45,4$ & 61,7 & $50,9-67,7$ \\
\hline$>\mathbf{8 0 0}$ Betten & 14 & 41,5 & $37,2-47,1$ & 59,9 & $53,4-67,9$ \\
\hline Universitätskliniken & 11 & 55,0 & $47,3-57,8$ & 78,5 & $71,4-82,8$ \\
\hline Gesamt & 109 & 43,5 & $36,0-47,9$ & 64,4 & $53,3-71,9$ \\
\hline
\end{tabular}

*RDD („recommended daily doses“ gemäß [8]) bzw. DDD gemäß ATC-WHO-Definition

teilnehmen und zu mindestens vier aufeinanderfolgenden Quartalen in den Jahren 2012-2013 vollständige Daten geliefert haben. Das ADKA-ifRKI-Surveillance-Projekt ist ein von der Abteilung Infektiologie des Universitätsklinikums Freiburg (if) entwickeltes Projekt, das seit 2008 kooperativ mit dem Bundesverband Deutscher Krankenhausapotheker (ADKA) weiterentwickelt wurde. Seit 2010 wird es in Zusammenarbeit mit dem Robert Koch-Institut (RKI) durchgeführt. Das Projekt hat inzwischen Teilnahmevereinbarungen mit>300 Akutkrankenhäusern (Stand 31.1.2015). Ein erster Vergleichsreport für Benchmarking-Zwecke wurde für das Jahr 2011 vorgelegt [7].

Im Projekt werden pro Quartal Verbrauchsdaten (Stückzahlen) zu allen systemischen Antiinfektiva (ATC: „anatomisch-therapeutisch-chemische Klassifikation“ Klasse J) zusammen mit den zugehörigen Belegungsdaten elektronisch eingelesen und kostenstellengenau ausgewertet. Die Daten aus dem Materialwirtschaftssystem der teilneh- menden Kliniken durchlaufen eine Qualitäts- und Plausibilitätsprüfung:

- korrekte Bezeichnung der Antiinfektiva

- Packungsgrößenangaben

- Kostenstellenplan-Zuordnungen und -änderungen

- starke Verbrauchsschwankungen gegenüber früheren Quartalen

Zur Analyse berechneten wir die Verbrauchsdichte (Tagesdosen pro 100 Pflegetage, minutengenaue Belegungsstatistik).

Als Tagesdosen werden primär krankenhausadaptierte, von uns in früheren Untersuchungen $[16,17]$ validierte Dosierungen verwendet („recommended daily doses“, RDD). Zudem berechneten wir auch definierte Tagesdosen („defined daily doses“ DDD) nach dem ATC-DDD-Index (Stand 1.1.2013) der WHO (Weltgesundheitsorganisation). DDD sind zwar international üblich, werden jedoch vor allem für vergleichende Analysen in der ambulanten Medizin als geeignet angesehen und verwendet.

\begin{tabular}{|c|c|c|c|c|}
\hline & \multicolumn{3}{|c|}{ RDD/100 } & \multirow[b]{2}{*}{$\begin{array}{l}\text { Anteil am } \\
\text { Gesamtverbrauch }\end{array}$} \\
\hline & $\mathbf{n}$ & Median & $\begin{array}{l}\text { Interquartil- } \\
\text { bereich }\end{array}$ & \\
\hline \multicolumn{5}{|l|}{ Nicht-universitäre Krankenhäuser } \\
\hline $\begin{array}{l}\text { Nicht-operative Abteilungen } \\
\text { (Normalstationen) }\end{array}$ & 316 & 39,0 & $31,7-46,1$ & $35 \%$ \\
\hline Hämatologie-Onkologie & 35 & 56,4 & $41,8-68,5$ & $5 \%$ \\
\hline Operative Abteilungen (Normalstationen) & 475 & 37,6 & $31,0-44,9$ & $48 \%$ \\
\hline Intensivstationen & 148 & 86,7 & $71,8-102,0$ & $12 \%$ \\
\hline \multicolumn{5}{|l|}{ Universitätskliniken } \\
\hline $\begin{array}{l}\text { Nicht-operative Abteilungen } \\
\text { (Normalstationen) }\end{array}$ & 91 & 44,0 & $40,9-48,7$ & $22 \%$ \\
\hline Hämatologie-Onkologie & 11 & 102,1 & $86,8-115,3$ & $12 \%$ \\
\hline Operative Abteilungen (Normalstationen) & 122 & 43,0 & $38,5-52,5$ & $48 \%$ \\
\hline Intensivstationen & 68 & 91,7 & $88,6-108,3$ & $18 \%$ \\
\hline
\end{tabular}

Tab. 2 Verbrauchsdichte systemisch wirksamer Antibiotika in verschiedenen Abteilungen / Abteilungsbereichen in Tagesdosen pro 100 Pflegetage. 
Zum Analysezeitpunkt hatten 143 Krankenhäuser Daten geliefert, darunter 122 komplette Verbrauchsdaten mit Pflegetagen zu mindestens einem Quartal 2012-2013 und 109 Krankenhäuser mit kompletten Daten zu mindestens vier aufeinanderfolgenden Quartalen.

Die systemisch antibakteriell aktiven Substanzen (ohne topisch wirksame Substanzen) der jeweils 4 jüngsten aufeinanderfolgenden Quartale pro Krankenhaus wurden für den vorliegenden Report deskriptiv unter Verwendung von Median und Interquartilbereich als Jahresdaten 2012/13 dargestellt. Es wurden alle Fachabteilungen außer Psychiatrie/Psychosomatik und Pädiatrie berücksichtigt. Als Vergleichsdaten haben wir die Ergebnisse aus einer älteren, methodisch ähnlichen Auswertung des Antibiotikaverbrauchs in deutschen Kliniken (Zahlen der Firma IMS Health) verwendet [5, 6].

\section{Ergebnisse}

Auswertbar für die jetzige Erhebung waren insgesamt 109 Krankenhäuser mit den jeweils jüngsten 4 aufeinanderfolgenden Quartalen während 2012/13 (76 mit Daten zum kompletten Jahr 2013) und einer Gesamtmenge von 14447298 Pflegeta- gen. Basierend auf der offiziellen Krankenhaus-Statistik für das Jahr 2012 entsprechen diese 6,4\% der Pflegetage in Akutkrankenhäusern in Deutschland.

Die Gruppe der teilnehmenden Krankenhäuser ist als Stichprobe nicht repräsentativ für die deutschen Akutkliniken. Es sind überproportional viele größere Krankenhäuser vertreten:

- Von den 1318 Krankenhäusern mit <400 Betten in Deutschland nahmen 50 teil (4\%),

- bei den Krankenhäusern mit 400-800 Betten lag dieser Anteil bei 34/282 (12\%),

- und bei den größeren Kliniken (>800 Betten) lag er bei 25/92 (27\%).

Die teilnehmenden Kliniken lieferten Daten zu 2555 Kostenstellen, die zu 1243 Fachabteilungen / Abteilungsbereichen zusammengefasst wurden, darunter insgesamt 35 hämatologisch-onkologische Abteilungen und 239 überwiegend (158/239) operative bzw. gemischt belegte Intensivstationen.

- 22 der teilnehmenden Krankenhäuser lagen in den neuen Bundesländern (inkl. Berlin),

- 48 im Süden (Baden-Württemberg plus Bayern), und

- unter den sonstigen Teilnehmern waren die meisten aus Nordrhein-Westfalen $(\triangleright$ Abb. 1$)$.
Abb. 2 Antibiotika-Verbrauchsdichte (in RDD/100) insgesamt und Verbrauchsdichte verschiedener Antibiotikaklassen 2012/13 in 109 Akutkrankenhäusern

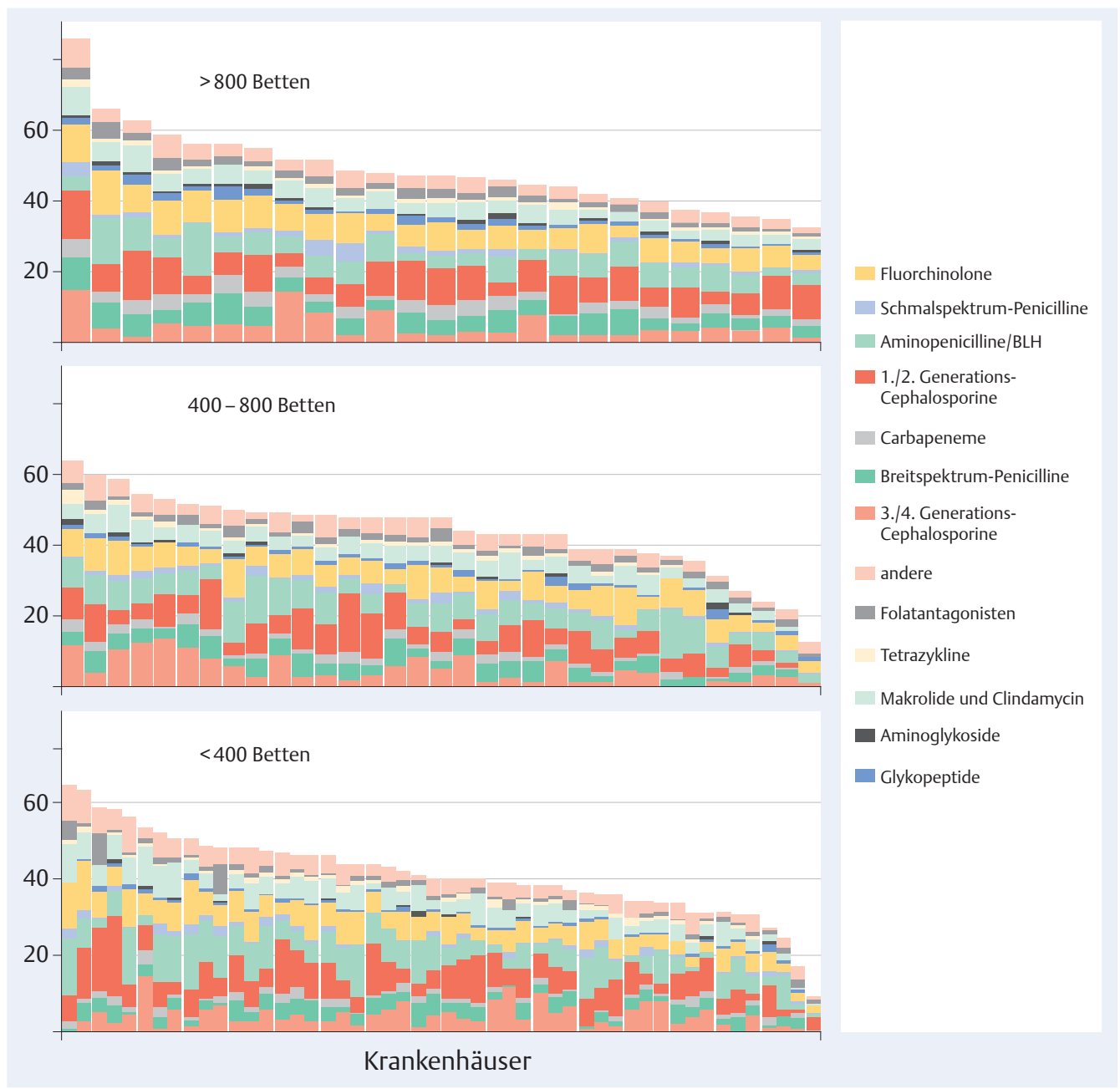




\begin{tabular}{|c|c|c|c|c|}
\hline & \multicolumn{2}{|c|}{$\begin{array}{l}\text { Nicht-universitäre } \\
\text { Krankenhäuser }\end{array}$} & \multicolumn{2}{|c|}{ Universitätskliniken } \\
\hline & Median & $\begin{array}{l}\text { Interquartil- } \\
\text { bereich }\end{array}$ & Median & $\begin{array}{l}\text { Interquartil- } \\
\text { bereich }\end{array}$ \\
\hline Carbapeneme & 1,4 & $1,0-2,3$ & 4,0 & $3,4-4,7$ \\
\hline Breitspektrum-Penicilline & 3,3 & $2,2-4,2$ & 6,1 & $4,2-6,4$ \\
\hline 3./4. Generations-Cephalosporine & 3,6 & $2,3-5,6$ & 4,1 & $2,8-5,4$ \\
\hline 1./2. Generations-Cephalosporine & 6,8 & $4,5-9,2$ & 9,4 & $6,0-10,4$ \\
\hline Aminopenicillin / BLI-Kombinationen ${ }^{1}$ & 6,1 & $4,0-7,9$ & 5,0 & $3,9-7,7$ \\
\hline Schmalspektrum-Penicilline ${ }^{2}$ & 1,3 & $0,9-1,7$ & 1,8 & $1,7-2,0$ \\
\hline Fluorchinolone & 5,5 & $4,1-7,0$ & 7,7 & $6,7-8,8$ \\
\hline Glykopeptide inkl. Daptomycin & 0,7 & $0,4-1,0$ & 2,1 & $1,7-2,3$ \\
\hline Aminoglykoside & 0,2 & $0,1-0,3$ & 0,5 & $0,4-0,8$ \\
\hline Makrolide und Clindamycin & 4,2 & $3,1-5,4$ & 4,9 & $4,2-5,5$ \\
\hline Tetrazykline & 0,5 & $0,3-0,7$ & 0,6 & $0,6-0,9$ \\
\hline Folatantagonisten / Sulfonamide & 1,2 & $0,9-1,6$ & 2,2 & $2,0-2,9$ \\
\hline andere & 3,7 & $2,7-4,8$ & 4,0 & $3,6-4,3$ \\
\hline darunter Metronidazol & 3,1 & $2,4-4,3$ & 2,5 & $2,1-3,0$ \\
\hline
\end{tabular}

Tab. 3 Verbrauchsdichte für bestimmte Antibiotikaklassen in Tagesdosen pro 100 Pflegetage (RDD/100).

Antibiotikagesamtverbrauch | Die AntibiotikaVerbrauchsdichte betrug insgesamt 43,5 RDD/100 Pflegetage (Median) mit einem Interquartilbereich von 36-48 RDD/100. In $>$ Tab. 1 sind die entsprechenden Ergebnisse je nach Krankenhausgröße/-art angegeben. Ebenso sind die Zahlen für den internationalen Vergleich in der Maßeinheit DDD/100 aufgelistet, mit der - auch gemäß anderen Erhebungen - die Verbrauchsdichte eher überschätzt wird [17-21].

Die Verbrauchsdichte war in Universitätskliniken signifikant höher ( $p<0,001$, Wilcoxon-Rangsummentest) als in den anderen Krankenhäusern (Median, 55 vs. 40,6 RDD/100), wohingegen keine Unterschiede zwischen den nicht-universitären Krankenhäusern unterschiedlicher Größe beobachtet wurden ( $\triangleright$ Tab. $\mathbf{1}$ )

Wie erwartet wurden die Antibiotika in den verschiedenen Fachabteilungen mit unterschiedlicher Intensität eingesetzt ( $\bullet$ Tab. 2). Besonders hoch war die Verbrauchsdichte in universitären hämatologisch-onkologischen Abteilungen (Median 102,1 RDD/100) sowie auf den Intensivstationen (Median, 86,7 bzw. 91,7 RDD/100 in nichtuniversitären Krankenhäusern bzw. Universitätskliniken). Auf den Intensivstationen war die Verbrauchsdichte rund doppelt so hoch wie auf den Normalstationen ( Tab. 2). Der Anteil der Antibiotika auf Intensivstationen am Gesamtverbrauch war jedoch sowohl in Universitätskliniken als auch in nicht-universitären Krankenhäusern $<20 \%$ ( Tab. 2$)$.

Antibiotikaklassen | Cephalosporine der ersten und zweiten Generation sowie Fluorchinolone waren die beiden am häufigsten eingesetzten Antibiotikaklassen. Breitspektrum-Betalaktame, darunter

- Carbapeneme,

- Piperacillin/Tazobactam sowie

- Cephalosporine der dritten und vierten Generation

wurden deutlich häufiger in Universitätskliniken verordnet $(\checkmark$ Tab. 3 ). Aber auch bei den meisten anderen Substanzen bzw. Substanzgruppen war die Verordnungsdichte in Universitätskliniken höher. Ein sehr deutlicher Unterschied bestand beim Verbrauch von Breitspektrum-Penicillinen (im wesentlichen Piperacillin-Tazobactam) und Carbapenemen. Aminoglykoside als auch Tetrazykline (inkl. Tigecyclin) wurden jeweils sehr wenig eingesetzt ( $<1$ RDD/100). Das Gleiche galt für Glyko- bzw. Lipopeptidantibiotika (inkl. Daptomycin) speziell an nicht-universitären Krankenhäusern.

- Abb. 2 zeigt die Variation und Verteilung der Gesamtverbrauchsdichte sowie der unterschiedlichen Anwendungsmuster und Präferenzen von eingesetzten Antibiotikaklassen in den einzelnen Kliniken. Bemerkenswert sind dabei vor allem die mit sehr unterschiedlicher Intensität verwendeten Cephalosporine und - korrespondierend - 


\begin{tabular}{lclc}
2004 & \multicolumn{2}{c}{$\mathbf{2 0 1 2 / 1 3}$} \\
Cefuroxim & $13,8 \%$ & Cefuroxim & $14,6 \%$ \\
Cotrimoxazol & $7,9 \%$ & Ampicillin-Sulbactam & $8,9 \%$ \\
Ampicillin-Sulbactam & $6,8 \%$ & Piperacillin-Tazobactam & $8,8 \%$ \\
Amoxicillin-Clavulansäure & $5,9 \%$ & Ceftriaxon & $8,2 \%$ \\
Ceftriaxon & $5,5 \%$ & Ciprofloxacin & $8,1 \%$
\end{tabular}

Tab. 4 Die 5 am häufigsten verordneten Substanzen (nach RDD) im Krankenhaus mit ihrem jeweiligen Anteil am Gesamtverbrauch (in \% RDD) 2004 und 2012/13.
Penicilline. Das Verhältnis zwischen Cephalosporinen und Penicillinen (in RDD) betrug insgesamt 51:49. Es schwankte zwischen 20:80 und 82:18.

Der Anteil von Cephalosporinen der ersten und zweiten Generation und der dritten und vierten Generation am Gesamtverbrauch je Krankenhaus schwankte von $5-37 \%$ bzw. von $<1-29 \%$. Die entsprechenden Verordnungsanteile von Fluorchinolonen bzw. Penicillinen schwankten von 6-27\% bzw. von 10-49\%. In operativen Abteilungen wurden Cephalosporine der ersten/zweiten Generation häufiger eingesetzt als in nicht-operativen Abteilungsbereichen: Die Medianwerte in den verschiedenen Krankenhausgruppen waren hier 9-13 RDD/100 für die operativen Fachbereichen im Vergleich zu 1,5-2,3 RDD/100 in den nicht-operativen Abteilungen.

Die fünf - gemessen an Tagesdosen (RDD) - am häufigsten eingesetzten Einzelsubstanzen waren

- Cefuroxim,

- Ampicillin-Sulbactam,

- Piperacillin-Tazobactam,

- Ceftriaxon und

- Ciprofloxacin ( Tab. 4).

Interessant ist die im Fall von Cefuroxim relativ häufig verordnete orale Form (Cefuroximaxetil), die in $53 \%$ aller verabreichten Cefuroxim-Tagesdosen (RDD) verwendet wurde. Zum Vergleich: Ampicillin-Sulbactam wurde (als Sultamicillin) in $36 \%$ der Tagesdosen oral verabreicht, Ciprofloxacin wurde in $70 \%$ der Tagesdosen oral verabreicht.

\section{Diskussion}

Erstmals liegen für eine größere Zahl von Akutkrankenhäusern in Deutschland zuverlässige und nicht unter dem Gesichtsprunkt der Marktforschung erhobene Antibiotikaverbrauchszahlen vor. Im Vergleich mit den Daten aus einer früheren Erhebung aus dem Jahre $2004[5,6]$ sind die jetzigen Zahlen höher. Der beobachtete Mehrverbrauch scheint sowohl die operative als auch konservative Medizin zu betreffen ( Abb. 3 ). Da es sich bei der jetzigen Untersuchung nicht um eine echte Längsschnitt-Studie handelt, sind genauere Angaben zu Veränderungen der Verbrauchsdichte über die Zeit nicht möglich.
Der geschätzte Anstieg des Antibiotikaverbrauchs (gemessen in RDD/100) innerhalb von knapp 10 Jahren (2004 bis 2012/13) würde je nach Klinikgröße eine Steigerung von 15\% (Universitätskliniken) bis $30 \%$ betragen. Betrachtet man Absolutwerte, betragen die geschätzten Änderungen in der Gesamtverbauchsdichte in dieser Periode für Krankenhäuser:

- <400 Betten: $31 \rightarrow 40$ RDD/100

- 400-800 Betten: $34 \rightarrow 42$ RDD/100

- nicht-universitäre Krankenhäuser mit >800 Betten: $36 \rightarrow 42$ RDD/100

- Universitätskliniken: $48 \rightarrow 55 \mathrm{RDD} / 100$

Dieser wahrscheinliche Anstieg dürfte zu einem großen Teil mit der Verkürzung der Verweildauer über diese Zeit und mit höherer Fallzahl und -komplexität erklärt werden. Repräsentative Punktprävalenzstudien (Verordnungsprävalenz an einem Stichtag) an deutschen Krankenhäusern haben zwischen 1994 und 2011 ebenfalls einen Anstieg in der Antibiotika-Anwendung um rund $32 \%$ beobachtet $[22,23]$. Auch wurde bei der Erhebung 2011 eine höhere Tagesprävalenz der Antibiotika-Anwendung in universitären als in nicht-universitären Krankenhäusern beschrieben (29,1 vs. $24,5 \%)$.

Trotz offenbar steigender Verbrauchsdichte liegt Deutschland aktuell im internationalen Vergleich jedoch nach wie vor nicht in der Spitzengruppe des Antibiotikaverbrauchs im Krankenhaus. Höhere Verbrauchsdichten wurden beispielsweise aus Dänemark und den Niederlanden berichtet, ähnliche aus Schweden und niedrigere aus Frankreich [3, 8, 24-28]. Zu berücksichtigen sind allerdings die Bettenkapazität insgesamt, die Verweildauer sowie Anteil tagesklinischer Behandlungen im Krankenhaus. Die Länder unterscheiden sich hier stark, was direkte Vergleiche erschwert.

Auch im ambulanten Bereich gibt es Unterschiede in der Antibiotika-Verbrauchsdichte. Diese ist beispielsweise in Frankreich sehr hoch, in den Niederlanden niedrig [3]. In Ländern wie Italien werden im ambulanten Bereich intensiv parenterale Antibiotika verordnet, was ebenfalls die Verbrauchsdichte in Kliniken beeinflussen dürfte.

Die auf Intensivstationen und vor allem in universitären hämatologisch-onkologischen Abteilungen deutlich höhere Antibiotika-Verbrauchsdichte ist nicht unerwartet und in anderen Untersuchungen wiederholt beobachtet worden [24-27]. Von Bedeutung ist, dass die Anteile des Antibiotikaverbrauchs in der Hämatologie-Onkologie und Intensivmedizin am Gesamtverbrauch einer Klinik in der Regel jedoch jeweils $<20 \%$ betragen und insofern eine geringere Beachtung der sonstigen Bereiche in Projekten zur Verbesserung der Verschreibungsqualität nicht rechtfertigt. 
Auffällig war in der aktuellen Analyse, dass Cefuroxim nach wie vor die am häufigsten verordnete Einzelsubstanz in der Klinik darstellt. Cefuroxim und andere Cephalosporine der ersten und zweiten Generation wurden in operativen Abteilungen häufig eingesetzt, und es ist bekannt, dass sie vielfach im Rahmen der perioperativen Prophylaxe - oft auch im Sinne einer nur selten indizierten postoperativ verlängerten Gabe - verwendet werden.

Der Anteil von Cephalosporinen war in einigen Kliniken sehr hoch. Intensiver Einsatz von Cephalosporinen, vor allem von Ceftriaxon, aber auch von Fluorchinolonen ist wiederholt mit hohen Raten von Clostridium-difficile-Infektionen (CDI), aber auch mit ESBL-produzierenden Gram-negativen Bakterien und Vancomycin-resistenten Enterokokken assoziiert worden [29-33]. Mehrere Studien haben durch Reduktion des Einsatzes von Cephalosporinen und/oder Fluorchinolonen die nosokomiale CDI-Rate senken können [29]. Studien aus Deutschland hierzu fehlen, und es wird von großem Interesse sein, die CDI-Häufigkeit mit bestimmten Antibiotikaverbrauchsmustern auch in deutschen Kliniken zu korrelieren und gegebenenfalls Antibiotikastrategie-Änderungen zu planen und zu evaluieren.

In Schweden hat man den Cephalosporin-Einsatz in Krankenhäusern zwischen 2006 und 2013 um $>50 \%$ gesenkt - inzwischen beträgt dort die Cephalosporinverbrauchsdichte nur noch rund 6 DDD/100 [28] - im Vergleich zu 16 (Mittelwert) bzw. 15 DDD/100 (Median) in unserer Erfassung. Eine ähnlich deutliche Verbrauchsreduktion vor allem bei Cephalosporinen der zweiten Generation wurde auch in England erzielt [34]. Ungewöhnlich ist auch die häufige Verordnung speziell von Cefuroximaxetil, das als Oral-Cephalosporin mit nur sehr mäßiger oraler Bioverfügbarkeit kaum für Indikationen bei Patienten im Akutklinikbereich geeignet erscheint. Hier sind detailliertere Verordnungsanalysen sinnvoll.

Fluorchinolone haben sich in Kliniken wie auch in der ambulanten Medizin zur zweitstärksten Antibiotikaklasse nach den Beta-Laktamen entwickelt [3]. Klinikweit schwankte ihre Verbrauchsdichte in der jetzigen Erhebung allerdings stark - zwischen $<1$ und $>12$ RDD/100 bzw. zwischen 6-27\% des Gesamtverbrauches. Extreme Schwankungen ( $<4$ bis $>30$ RDD/100) fanden sich speziell in hämatologisch-onkologischen Abteilungen, aber auch auf den Intensivstationen ( $<1$ bis $>50$ RDD/100), was auf sehr unterschiedliche lokale Behandlungsleitlinien bzw. deren Umsetzung hindeutet.

Ursachen hierfür und die Folgen für Behandlungsergebnis und Resistenz-Entwicklung sind nicht klar. Intensiver Fluorchinoloneinsatz in Kliniken ist nicht nur mit CDI, sondern wiederholt auch mit erhöhten MRSA-Raten assoziiert worden [35-37], und ihr Einsatz bei Harnwegsinfektionen und intraabdominellen Infektionen wird wegen der bei Escherichia coli beobachteten Resistenzraten von $>20 \%$ als problematisch angesehen. Aminoglykoside, eine Alternative zu Fluorchinolonen, bei denen die Resistenzraten in Deutschland bei vielen relevanten Infektionserregern gering sind, werden im Klinikbereich kaum noch eingesetzt.

Die Antibiotika-Verbrauchsdichte in dieser Erhebung unterschied sich klinikweit um den Faktor 1,4 (Universitätskliniken) bis 7,5 (Krankenhäuser mit<400 Betten). Sehr deutliche Schwankungen finden sich teilweise auch bei Betrachtung von Fachbereichen. Dies weist auf die Schwierigkeiten der Bewertung von Verbrauchsmengen bzw. Verbrauchsdichten hin. Die Berücksichtigung von Krankenhausgröße und die Nicht-Berücksichtigung von psychiatrischen und pädiatrischen Abteilungen in dieser Erhebung ist unserer Meinung nach noch nicht ausreichend als Adjustierung.

Es sind zahlreiche weitere Variablen bei einer detaillierteren Bewertung zu beachten, z. B.:

- Behandlungsschwerpunkte

- Abteilungsstrukturen

- Inanspruchnahme

- Belegungsstatistiken

- Krankenhausträger

- Verfügbarkeit von infektiologischen Fachabteilungen und Antibiotic Stewardship-Teams

Nicht nur hämatologisch-onkologische Abteilungen sollten separat dargestellt werden. Auch eine Betrachtung von neurologischen Abteilungen separat von internistischen Abteilungen und die getrennte Auswertung von operativen Fachabteilungen (z.B. Urologie oder HNO oder Orthopädie) sind sinnvoll und notwendig.

Derartige Vergleiche bieten sich bei sachgerechter Interpretation als Benchmarking und zur internen Qualitätssicherung an [27, 38]. Sie werden von

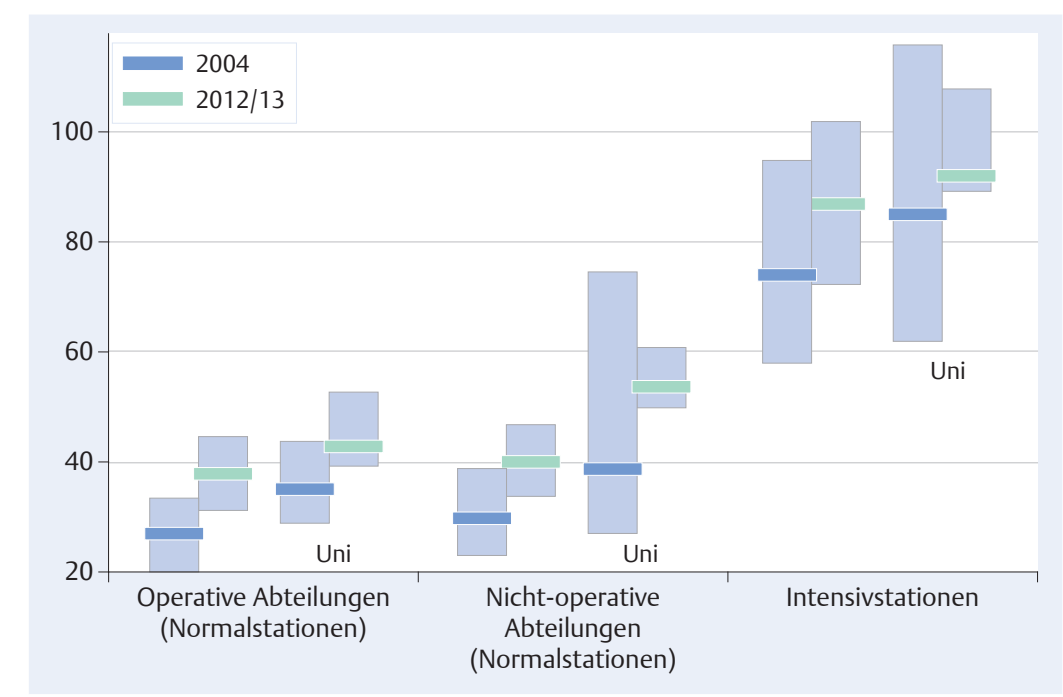

Abb. 3 Antibiotika-Verbrauchsdichte (in RDD/100) insgesamt 2012/13 im Vergleich zu 2004. Gezeigt sind jeweils die Mediane und Interquartilbereiche für verschiedene Fachabteilungsruppen in nicht-universitären Krankenhäusern und
Universitätskliniken („Uni“). 
uns bereits jetzt den teilnehmenden Krankenhäusern angeboten, werden jedoch für die selteneren Fachabteilungen erst mit einer größeren Zahl von teilnehmenden Kliniken ausreichend zuverlässig sein. Weitere Adjustierungen an patientenspezifische Variablen sowie eine Erfassung von Indikationen (z.B. therapeutischer oder prophylaktischer Einsatz, ambulant oder nosokomial erworbene Infektion) werden dann möglich und sinnvoll werden, wenn die Verbrauchsmengenerhebung über moderne Krankenhausinformationssysteme individuell erfolgen kann.

Von großer Bedeutung ist die Verwendung realistischer Tagesdosendefinitionen. Bewährt hat sich dabei, anstelle (oder als Zusatz zu) den von der WHO definierten DDD an das Krankenhaus adaptierte Definitionen (wie RDD in dieser Untersuchung) zu wählen. Deren Vorteil besteht vor allem darin, dass die Definitionen der Tagesdosen von Penicillinen sehr viel näher die tatsächlichen Dosierungen abbilden, und es so weniger zu Verzerrungen bei Kliniken oder Abteilungen mit intensivem Einsatz von Penicillinen kommt [16-21]. Vor allem Kliniken mit einer Präferenz für Penicilline (z. B. gegenüber Cephalosporinen) können bei (alleiniger) Verwendung von WHO-DDD fälschlicherweise als Hochverbraucherkliniken eingeordnet werden. Die Erfassung von tatsächlich verordneten Tagesdosen mittels Punktprävalenzuntersuchung oder besser über elektronische Krankenaktenauswertung könnte helfen diese Limitation zu vermeiden $[38,39]$.

Einschränkungen der aktuellen Analyse sind

- die immer noch begrenzte Zahl der teilnehmenden Kliniken,

- die noch nicht ausreichende Repräsentativität und

- die bisher fehlende Längsschnittbetrachtung derselben Kliniken über einige Jahre.

Mit besserer Infrastruktur und Expertise in den einzelnen Kliniken und modernsten Schnittstellen und Unterstützung bei der Datenerfassung und Übertragung werden jedoch solche Daten verlässlicher, kontinuierlich und in geeigneter Form verfügbar und besser interpretierbar sein.

Mit der letzten Änderung des Infektionsschutzgesetzes ist eine klinikinterne Erfassung und Bewertung des Antiinfektivaeinsatzes verpflichtend geworden. Hierzu wurden Empfehlungen konsentiert, in die unsere Erfahrungen mit der Antiinfektiva-Surveillance im Rahmen der internen Qualitätssicherung und zum Zweck der Sicherstellung einer rationalen Verschreibungspraxis eingeflossen sind [40]. Die vergleichende Auswertung von Antibiotikaverbrauchsmustern in Akutkliniken mit Rückmeldung und fachbereichsorientiertem Benchmarking kann unserer Meinung nach wichtige Anstöße zur Optimierung des AntibiotikaEinsatzes und Planung und Beurteilung von Strategien zur Resistenzminimierung geben.

\section{Konsequenz für Klinik und Praxis}

- Die Antibiotika-Verbrauchsdichte in deutschen Akutkrankenhäusern im Jahr 2012/13 betrug 43,5 Tagesdosen pro 100 Belegungstage und war in Universitätskrankenhäusern höher als in nichtuniversitäten Kliniken - dies entspricht einem Anstieg um 15-30\% in den letzten 10 Jahren.

- Cephalosporine wurden etwas häufiger als Penicilline eingesetzt. Fluorchinolone machten nach Cephalosporinen und Penicillinen die drittstärkste Substanzklasse aus. Aminoglykoside werden kaum noch verwendet. Die am häufigsten eingesetzte Einzelsubstanz war Cefuroxim.

- Erfassungen und Analysen wie diese können Anstöße zur Optimierung des Antibiotika-Einsatzes sowie zur Planung und Beurteilung von Strategien zur Resistenzminimierung, Eindämmung von Clostridium-difficile-Infektionen und zur Verbesserung der Patientensicherheit geben.

\section{Danksagung}

Die Studie wurde finanziell teilweise unterstützt durch den Bundesverband Deutscher Krankenhausapotheker (ADKA), die Deutsche Gesellschaft für Infektiologie (DGI), das Robert Koch-Institut (RKI) sowie Forschungsbeihilfen an WVK. des BMBF und der EU. Wir danken Nadine Weber, Antonia Hug, Sven-Ulrich Steib und Lin Wang für die Hilfe bei der Datenerfassung und Marcel Feig für die Hilfe bei der Erstellung individueller Antiinfektivareports. Die Untersuchung wäre ohne die freiwillige Teilnahme vieler deutscher Krankenhäuser nicht möglich gewesen, für die wir uns hiermit bedanken.

\section{Interessenkonflikte}

KdW ist derzeitige Sprecherin der DGI (Deutsche Gesellschaft für Infektiologie)-Sektion Antibiotic Stewardship sowie stellvertretende Sprecherin der Kommission „Antibiotika, Resistenz und Therapie“ beim Robert Koch-Institut.

MF ist derzeit Sprecher des ADKA-Ausschusses Antiinfektive Therapie. Er erhielt Honorare für wissenschaftliche Vorträge von Amgen, Astellas, Astra-Zeneca, Fresenius-Kabi und Pfizer.

$\mathrm{MH}$ erhielt Honorare für wissenschaftliche Vorträge von Astellas, B. Braun, Pfizer, Roche und Teva.

THT erhielt Forschungsbeihilfen und Honorare für wissenschaftliche Vorträge von B. Braun, Novartis, Otsuka, Sanofi und Teva.

WVK erhielt Forschungsbeihilfen, Reisekostenerstattung oder Honorare für wissenschaftliche Vorträge von Bayer, BMG, BMBF, Boehringer, DFG, DGI, EU, ESCMID, Infectopharm, Gilead, GSK, Janssen, Pfizer, RKI, Sobi und Stiftung Warentest.

GF und MSB erklären, dass kein Interessenkonflikt besteht.

\section{Literatur}

1 Michael CA, Dominey-Howes D, Labbate M. The antimicrobial resistance crisis: causes, consequences, and management. Front Public Health 2014; 2: 145 
2 You Y, Silbergeld EK. Learning from agriculture: understanding low-dose antimicrobials as drivers of resistome expansion. Front Microbiol 2014; 5 : 284

3 European Centre for Disease Prevention and Control. Surveillance of antimicrobial consumption in Europe 2012. Stockholm: ECDC; 2014. http://ecdc.europa.eu/en/publications/_layouts/ forms/Publication_DispForm. aspx?List $=4$ f55ad51 4aed-4d32-b960-af70113dbb90\&ID =1174 Letzter Zugriff am 27.10.2015

$4 E C D C / E F S A / E M A$. First joint report on the integrated analysis of the consumption of antimicrobial agents and occurrence of antimicrobial resistance in bacteria from humans and food-producing animals - Joint Interagency Antimicrobial Consumption and Resistance Analysis (JIACRA).

http://www.ema.europa.eu/docs/en_GB/ document_library/Report/2015/01/

WC500181485.pdf

Letzter Zugriff am 27.10.2015

5 Bundesamt für Verbraucherschutz und Lebensmittelsicherheit, Hrsg. GERMAP 2008 - AntibiotikaResistenz und -Verbrauch. http://www.bvl.bund.de/SharedDocs/Downloads/08_Presselnfothek/Germap_2008. pdf? blob=publicationFile \& $v=2$ Letzter Zugriff am 27.10.2015

6 Bundesamt für Verbraucherschutz und Lebensmittelsicherheit, Hrsg. GERMAP 2010 - AntibiotikaResistenz und -Verbrauch http://www.bvl.bund.de/SharedDocs/Downloads/08_Presselnfothek/Germap_2010. pdf?_blob=publicationFile $\& v=2$ Letzter Zugriff am 27.10.2015

7 Bundesamt für Verbraucherschutz und Lebensmittelsicherheit, Hrsg. GERMAP 2012 - AntibiotikaResistenz und -Verbrauch. http://www.bvl.bund.de/SharedDocs/Downloads/05_Tierarzneimittel/germap2012. pdf? blob=publicationFile \& $v=4$ Letzter Zugriff am 27.10.2015

8 Statens Serum Institut, National Veterinary Institute, Technical University of Denmark, National Food Institute, Technical University of Denmark, Hrsg. DANMAP 2013. Use of antimicrobial agents and occurrence of antimicrobial resistance in bacteria from food animals, food and humans in Denmark.

http://www.danmap.org/ /media/Projekt\%20 sites/Danmap/DANMAP\%20reports/DANMAP\%20 2013/DANMAP\%202013.ashx Letzter Zugriff am 27.10.2015

9 Vander Stichele RH, Elseviers MM, Ferech $\mathrm{M}$ et al. Hospital consumption of antibiotics in 15 European countries: results of the ESAC Retrospective Data Collection (1997-2002). J Antimicrob Chemother 2006; 58: 159-167

10 van Boeckel TP, Gandra S, Ashok A et al. Global antibiotic consumption 2000 to 2010: an analysis of national pharmaceutical sales data. Lancet Infect Dis 2014; 14: 742-750

11 van Kleef E, Robotham JV, Jit M et al. Modelling the transmission of healthcare associated infections: a systematic review. BMC Infect Dis 2013; 13: 294

12 Davis GS, Sevdalis N, Drumright LN. Spatial and temporal analyses to investigate infectious disease transmission within healthcare settings. J Hosp Infect 2014; 86: 227-243

13 Deutschen Gesellschaft für Infektiologie. S3-Leitinie Strategien zur Sicherung rationaler Antibiotika-Anwendung im Krankenhaus - AWMFRegisternummer 092/001.

http://www.awmf.org/uploads/tx_szleitlinien/092001I_S3_Antibiotika_Anwendung_im_
Krankenhaus_2013-12.pdf

Letzter Zugriff am 27.10.2015

14 Johannsson B, Beekmann SE, Srinivasan A et al. Improving antimicrobial stewardship: the evolution of programmatic strategies and barriers. Infect Control Hosp Epidemiol 2011; 32: 367-374

15 Hamilton KW, Fishman NO. Antimicrobial stewardship interventions: thinking inside and outside the box. Infect Dis Clin North Am 2014; 28: 301-313

16 de With K, Meyer E, Steib-Bauert M et al. Antibiotic use in two cohorts of German intensive care units. J Hosp Infect 2006; 64: 231-237

17 de With K, Bestehorn H, Steib-Bauert M, Kern WV. Comparison of defined versus recommended versus prescribed daily doses for measuring hospital antibiotic consumption. Infection 2009; 37: 349-352

18 Muller A, Monnet DL, Talon D et al. Discrepancies between prescribed daily doses and WHO defined daily doses of antibacterials at a university hospital. Br J Clin Pharmacol 2006; 61: 585-591

19 Mandy B, Koutny E, Cornette C et al. Methodological validation of monitoring indicators of antibiotics use in hospitals. Pharm World Sci 2004; 26: 90-95

20 Gagliotti C, Ricchizzi E, Buttazzi R et al. Hospital statistics for antibiotics: defined versus prescribed daily dose. Infection 2014; 42: 869-873

21 Haug JB, Reikvam Å. WHO defined daily doses versus hospital-adjusted defined daily doses: impact on results of antibiotic use surveillance. J Antimicrob Chemother 2013; 68: 2940-2947

22 Behnke M, Hansen S, Leistner R et al. Nosocomial infection and antibiotic use: a second national prevalence study in Germany. Dtsch Arztebl Int 2013; 110: 627-633

23 Hansen S, Sohr D, Piening B et al. Antibiotic usage in German hospitals: results of the second national prevalence study. J Antimicrob Chemother 2013; 68: 2934-2939

24 NethMap 2014 - Consumption of antimicrobial agents and antimicrobial resistance among medically important bacteria in The Netherlands in 2013.

http://www.swab.nl/swab/cms3.nsf/uploads/05AB E3EF93A82F4BC1257D07001DE8BC/\$FILE/ Boek\%20Nethmap-MARAN\%202014\%20TG.pdf Letzter Zugriff am 27.10.2015

25 Dumartin C, L'Hériteau F, Péfau M et al. Antibiotic use in 530 French hospitals: results from a surveillance network at hospital and ward levels in 2007. J Antimicrob Chemother 2010; 65: 2028-2036

26 Dumartin C, Rogues AM, L'Hériteau F et al. Consommation d'antibiotiques dans les établissements de santé français, réseau ATB-Raisin, 2008-2010. Bulletin Epidémiologique Hebdomadaire 2012; 42-43: 486-490

27 Institut de veille sanitaire. Surveillance de la consommation des antibiotiques ATB-Raisin - Synthèse des données 2012.

http://www.invs.sante.fr/content/ download/87329/320425/version/3/file/Rapport surveillance_consommation_antibiotiques_atb_ raisin_2012.pdf

Letzter Zugriff am 27.10.2015

28 SWEDRES-SVARM 2013. Use of antimicrobials and occurrence of antimicrobial resistance in Sweden. http://www.sva.se/globalassets/redesign2011/ pdf/om_sva/publikationer/swedres_svarm2013. $\mathrm{pdf}$ Letzter Zugriff am 2.11.2015

29 Feazel LM, Malhotra A, Perencevich EN et al. Effect of antibiotic stewardship programmes on Clostridium difficile incidence: a systematic review 
and meta-analysis. J Antimicrob Chemother 2014; 69: $1748-1754$

30 Gbaguidi-Haore H, Dumartin C, L'Hériteau F et al. Antibiotics involved in the occurrence of antibioticresistant bacteria: a nationwide multilevel study suggests differences within antibiotic classes. J Antimicrob Chemother 2013; 68: 461-470

31 McKinnell JA, Kunz DF, Chamot E et al. Association between vancomycin-resistant Enterococci bacteremia and ceftriaxone usage. Infect Control Hosp Epidemiol 2012; 33: 718-724

32 Hayakawa K, Marchaim D, Palla M et al. Epidemiology of vancomycin-resistant Enterococcus faecalis: a case-case-control study. Antimicrob Agents Chemother 2013; 57: 49-55

33 Pereira JB, Farragher TM, Tully MP, Cooke J. Association between Clostridium difficile infection and antimicrobial usage in a large group of English hospitals. Br J Clin Pharmacol 2014; 77: 896-903

34 Cooke J, Stephens P, Ashiru-Oredope D et al. Longitudinal trends and cross-sectional analysis of English national hospital antibacterial use over 5 years (2008-13): working towards hospital prescribing quality measures. | Antimicrob Chemother 2015; 70: 279-285
35 Weber SG, Gold HS, Hooper DC et al. Fluoroquinolones and the risk for methicillin-resistant Staphylococcus aureus in hospitalized patients. Emerg Infect Dis 2003; 9: 1415-1422

36 LeBlanc L, Pépin J, Toulouse K et al. Fluoroquinolones and risk for methicillin-resistant Staphylococcus aureus, Canada. Emerg Infect Dis 2006; 12: 1398-1405

37 Schneider-Lindner V, Delaney JA, Dial S et al. Antimicrobial drugs and community-acquired methicillin-resistant Staphylococcus aureus, United Kingdom. Emerg Infect Dis 2007; 13 : 994-1000

38 Boussat S, Demoré B, Lozniewski A et al. How to improve the collection and analysis of hospital antibiotic consumption: preliminary results of the ConsoRes software experimental implementation. Med Mal Infect 2012; 42: 154-160

39 Gravatt LA, Pakyz AL. Challenges in measuring antibiotic consumption. Curr Infect Dis Rep 2013; 15: 559-563

40 Schweickert B, Kern WV, de With K et al. Antibiotika-Verbrauchs-Surveillance. Bundesgesundheitsblatt Gesundheitsforschung Gesundheitsschutz 2013; 56: 903-912
DOI 10.1055/s-0041-105938

Dtsch Med Wochenschr 2015; 140: e237-e246

(c) Georg Thieme Verlag KG .

Stuttgart · New York.

ISSN 0012-0472

\section{License terms}

